\title{
Pediatric Neuroblastoma with Brain Metastasis: The Prognostic Role of Surgery
}

\author{
Melis GULTEKIN ${ }^{1}$, Caglayan Selenge BEDUK ESEN ${ }^{1}$, Ali VARAN² ${ }^{2}$, Canan AKYUZ², Burcak BILGINER ${ }^{3}$, \\ Ferah YILDIZ ${ }^{1}$, Murat GURKAYNAK ${ }^{1}$ \\ ${ }^{1}$ Hacettepe University, Faculty of Medicine, Department of Radiation Oncology, Ankara, Turkey \\ ${ }^{2}$ Hacettepe University, Faculty of Medicine, Department of Pediatric Oncology, Ankara, Turkey \\ ${ }^{3}$ Hacettepe University, Faculty of Medicine, Department of Neurosurgery, Ankara, Turkey \\ This study has been presented at the $23^{\text {rd }}$ National Cancer Congress of Turkish Society for Radiation Oncology between 17 and 21 April 2019 at Antalya, \\ Turkey
}

Corresponding author: Melis GULTEKIN melisbahadir@yahoo.com

\section{ABSTRACT}

AIM: To analyze the prognostic factors and treatment outcomes of neuroblastoma patients with brain metastasis.

MATERIAL and METHODS: The medical records of 7 patients with brain metastasis among 120 neuroblastoma patients treated with radiotherapy between June 2002 and December 2018 at our department were collected retrospectively. Survival time and prognostic factors were evaluated.

RESULTS: The prevalence of brain metastasis was 7/120 (5.8\%). The median age was 35 months (25-64). Three (43\%) patients had single brain metastasis. Two (29\%) patients with solitary metastases underwent gross total resection and 1 (14\%) patient with two lesions underwent subtotal resection for brain metastasis. All patients received chemotherapy and radiotherapy. The median time interval between the initial diagnosis and the brain metastasis was 14 months (0-28 months) in all patients; 12 months (0-28 months) in deceased patients and 23 months (19-26 months) in patients who were still alive at the time of this analysis ( $p=0.245)$. The median overall survival time after the treatment of brain metastasis was 14 months (7-22 months). Five patients died from progressive disease and 2 patients who had gross total resection were alive for 164 and 187 months, respectively. While the 5-year overall survival rate was $67 \%$ in patients with isolated brain metastasis, the 5 -year overall survival rate was $0 \%$ in the presence of other metastases $(\mathrm{p}=0.221)$.

CONCLUSION: Isolated brain metastasis and gross total resection of the brain metastasis seems to be favorable prognostic factors. Patients with solitary brain metastasis should be consulted for brain surgery.

KEYWORDS: Neuroblastoma, Brain metastasis, Surgery, Treatment, Radiotherapy, Prognosis

ABBREVIATIONS: CT: Computed tomography, MIBG: Metaiodobenzylguanidine, MRI: Magnetic resonance imaging, LDH: Lactate dehydrogenase

\section{INTRODUCTION}

$\mathrm{N}$ euroblastoma is the most common extracranial solid malignancy in children that is often diagnosed during the first year of life; it has an incidence of $7.4 \%$ in Turkey
(1). It has a varied clinical behavior, ranging from spontaneous regression to an aggressive clinical course, resulting in metastasis, and is responsible for $15 \%$ of childhood cancer mortality (15).

\begin{tabular}{llll}
\hline Melis GULTEKIN & (D) : 0000-0002-1806-2619 & Canan AKYUZ & (D) : 0000-0002-7105-4191 \\
$\begin{array}{llll}\text { C. Selenge BEDUK ESEN } & \text { (D) : 0000-0001-9967-8177 } & \text { Burcak BILGINER } & \text { (D) : 0000-0001-9667-3709 } \\
\text { Ali VARAN } & \text { (D) : } 0000-0003-4911-1476 & \text { Ferah YILDIZ } & \text { (D) : 0000-0002-2557-8103 }\end{array}$
\end{tabular}


At the time of diagnosis, more than $50 \%$ of patients present with metastatic disease and have a poor prognosis (13). The most common sites of metastases are the bone, bone marrow, and liver. Although brain metastases resulting from the extension of bone metastases of the skull are common, isolated parenchymal brain metastasis is rare, with a reported incidence ranging from $1.7 \%$ to $25 \%$ in patients with neuroblastoma $(2,4,17,22)$. Since survival rates in neuroblastoma are prolonged owing to aggressive chemotherapy, surgery, radiotherapy, autologous stem cell transplantation, and immunotherapy, the probability of brain metastases increases during the course of the disease $(4,13,21)$. Patients with brain metastasis have a poor prognosis, and there is no consensus on the optimal treatment for these patients $(5,11,18)$. Although brain metastases in these patients frequently result in death, long-term survival has been reported in selected cases with multidisciplinary treatment $(10,22)$. Due to its rarity, there are a limited number of studies in the literature $(4,9-11,13,16,18,22)$. Therefore, we aimed to analyze the prognostic features and treatment outcomes of neuroblastoma patients with brain metastases.

\section{MATERIAL and METHODS}

\section{Patients}

The medical records of seven patients with brain metastasis among 120 neuroblastoma patients treated with adjuvant and/or palliative radiotherapy between June 2002 and December 2018 at our department were collected retrospectively. Patients who were younger than 18 years and had parenchymal brain metastasis at the time of diagnosis or developed brain metastasis after initial treatment were included in this study. Patients with isolated cranial bone involvement and/or extension from dural, epidural, or skull metastases were excluded. This retrospective study was conducted in accordance with the principles of the Declaration of Helsinki. Ethical approval for this study was obtained from the institutional review board.

\section{Staging}

At the time of initial diagnosis, chest and abdominal computed tomography (CT) scans were performed in three patients, and metaiodobenzylguanidine (MIBG) scan and abdominal magnetic resonance imaging (MRI) scan were performed in four patients. Bone marrow biopsy, skeletal survey, and urine catecholamine analyses were performed in all patients. Lumbar puncture was not performed in any patient at the time of diagnosis. The diagnosis was histologically confirmed by surgery or biopsy. Patients were classified according to the International Neuroblastoma Staging System (6).

\section{First-Line Treatment}

All children had stage IV and high-risk disease at the initial diagnosis. Patients were treated with a risk-based prospective national neuroblastoma treatment protocol (TPOG-NBL2003 and TPOG-NBL2009) (1,14). In brief, all high-risk patients were treated with conventional chemotherapy, high-dose chemotherapy, and autologous stem cell transplantation. The conventional chemotherapy arm included three to four cycles of intensified induction chemotherapy ( $A 3$ and $A 5$ regimens in TPOG-NBL2003 or A9 and A11 regimens in TPOG-NBL2009). While the $A 3$ and $A 9$ regimens included vincristine, ifosfamide, dacarbazine, and adriamycin, the $A 5$ and $A 11$ regimens included cisplatin, cyclophosphamide, and etoposide. Surgery was performed at diagnosis or after induction chemotherapy. After surgery, cyclophosphamide maintenance therapy was continued. If patients had a residual viable tumor or a positive surgical margin, adjuvant radiotherapy was applied to the primary tumor site. Six cycles of 13-cis-retinoic acid were administered as maintenance therapy.

\section{Brain Metastasis}

The clinical symptoms of seven patients with brain metastasis included headache, vomiting, visual loss, lower limb motor function impairment, and seizures. Since these symptoms suggested brain metastases, contrast-enhanced brain MRI was performed for all patients, and salvage therapy, including surgery, radiotherapy, and chemotherapy, was administered. When patients had single brain metastasis with controlled primary tumor or symptoms of increased intracranial pressure, gross total resection or subtotal resection was performed. Histopathological confirmation was also obtained if it was possible to perform surgery. Craniospinal irradiation was performed in patients with leptomeningeal involvement of both the brain and spine, while local or whole-brain radiotherapy was applied to all other patients. If patients had symptoms related to brain metastasis, steroid treatment and/or antiepileptics were also administered.

\section{Follow-up}

All patients underwent routine follow-up every 3 months for the first 2 years, every 6 months for years 3-5, and then annually. Local and distant recurrences were assessed at each followup visit. Treatment toxicities were assessed according to the Radiation Therapy Oncology Group/European Organization for the Treatment of Cancer toxicity criteria (7).

\section{Statistical Analysis}

The impact of age, sex, symptoms of brain metastases, lactate dehydrogenase (LDH) level at diagnosis, number of brain metastases, location of brain metastases, other metastatic sites, the time interval between initial diagnosis and brain metastasis on overall survival, and treatment type before and after brain metastases were evaluated. Descriptive data were presented using medians and ranges for nonnormally distributed and ordinal variables. The overall followup was calculated from the time of diagnosis to death or the last follow-up. The follow-up time after radiotherapy for brain metastasis was also calculated as the period between the last day of radiotherapy and death or the last follow-up. Kaplan-Meier survival estimates were calculated. All statistical analyses were performed using SPSS version 21.0 software (SPSS Inc., Chicago, IL, USA). 


\section{RESULTS}

\section{Clinical Characteristics of Initial Diagnosis}

Patient, tumor, and treatment characteristics are presented in Table I. There were four girls and three boys with a median age of 35 months (range, 25-64 months) at the time of primary tumor diagnosis. The primary tumor was in the adrenal gland in six (86\%) patients and the para-spinal region in one (14\%) patient (patient 3). All patients were diagnosed with stage IV disease at the time of diagnosis. While bone metastasis was observed in all patients, additional bone marrow involvement was observed in four patients (patients 2-6), liver metastasis was observed in two patients (patients 5 and 6), and both lung metastasis and leptomeningeal involvement were observed in one patient (patient 6). The median LDH level was $1002 \mathrm{U} / \mathrm{mL}$ (range, 394-4321 U/mL) at the time of diagnosis. The LDH level was higher than $1500 \mathrm{U} / \mathrm{mL}$ in two patients (patients 6 and 7). N-Myc amplification was negative in two patients, positive in one patient, and unknown in four patients.
All but one patient were initially treated with induction chemotherapy according to the TPOG-NBL2003 and TPOGNBL2009 protocols. However, one (14\%) patient who had leptomeningeal involvement in both the brain and spine at the time of diagnosis was first treated with craniospinal irradiation followed by induction chemotherapy. After induction chemotherapy, all patients underwent surgery followed by radiotherapy at the primary tumor site. Only one patient underwent autologous stem cell transplantation (patient 3).

The median follow-up time was 30 months (range, 7-213 months) after initial diagnosis. The 2- and 5-year overall survival rates were $57 \%$ and $29 \%$, respectively. The median overall survival time was $30 \pm 14$ months (range, 2-58 months; 95\% Cl).

\section{Clinical Characteristics of Brain Metastasis and Outcomes}

The clinical characteristics, treatment features, and outcomes of neuroblastoma patients with brain metastases are shown in Table II. The prevalence of brain metastasis in this study was

Table I: Patient, Tumor, and Treatment Characteristics at the Time of Initial Diagnosis

\begin{tabular}{|c|c|c|c|c|c|c|c|c|}
\hline $\begin{array}{l}\text { Patient } \\
\text { no. }\end{array}$ & $\begin{array}{c}\text { Age } \\
\text { (months) }\end{array}$ & $\begin{array}{c}\text { Sites of } \\
\text { metastases }\end{array}$ & $\begin{array}{c}\text { LDH } \\
\text { level } \\
\text { (U/mL) }\end{array}$ & Chemo* & $\begin{array}{l}\text { Surgery } \\
\text { for the } \\
\text { primary } \\
\text { site }\end{array}$ & RT & $\begin{array}{c}\text { Time duration } \\
\text { to the } \\
\text { diagnosis } \\
\text { of brain } \\
\text { metastasis } \\
\text { (months) }\end{array}$ & $\begin{array}{l}\text { Other sites of } \\
\text { metastases } \\
\text { at the time } \\
\text { of brain } \\
\text { metastases }\end{array}$ \\
\hline 1 & 30 & Bone & 1002 & $\begin{array}{c}\mathrm{A} 3 \text { and } \mathrm{A} 5 \\
\text { regimens }\end{array}$ & GTR & $\begin{array}{c}1.5 \mathrm{~Gy} / 30 \mathrm{~Gy} \\
\text { to the tumor } \\
\text { bed }\end{array}$ & 19 & None \\
\hline 2 & 53 & Bone, BM & 549 & $\begin{array}{c}\mathrm{A} 3 \text { and } \mathrm{A} 5 \\
\text { regimens }\end{array}$ & GTR & $\begin{array}{c}1.5 \mathrm{~Gy} / 30 \mathrm{~Gy} \\
\text { to the tumor } \\
\text { bed }\end{array}$ & 26 & None \\
\hline 3 & 64 & Bone, BM & 1208 & $\begin{array}{l}\text { A3 and A5 } \\
\text { regimens }\end{array}$ & GTR & $\begin{array}{l}1.5 \mathrm{~Gy} / 30 \mathrm{~Gy} \\
\text { to the tumor } \\
\text { bed }\end{array}$ & 12 & None \\
\hline 4 & 35 & Bone, BM & 394 & $\begin{array}{c}\mathrm{A} 9 \text { and } \mathrm{A} 11 \\
\text { regimens }\end{array}$ & GTR & $\begin{array}{c}1.8 \mathrm{~Gy} / 21.6 \mathrm{~Gy} \\
\text { to the tumor } \\
\text { bed }\end{array}$ & 28 & Bone \\
\hline 5 & 35 & $\begin{array}{c}\text { Bone, BM, } \\
\text { liver }\end{array}$ & 604 & $\begin{array}{c}\mathrm{A} 9 \text { and } \mathrm{A} 11 \\
\text { regimens }\end{array}$ & GTR & $\begin{array}{c}1.8 \mathrm{~Gy} / 25.2 \mathrm{~Gy} \\
\text { to the tumor } \\
\text { bed }\end{array}$ & 4 & Bone \\
\hline 6 & 59 & $\begin{array}{c}\text { Bone, BM, } \\
\text { liver, lung, } \\
\text { LMI }\end{array}$ & 2069 & $\begin{array}{c}\mathrm{A} 9 \text { and } \mathrm{A} 11 \\
\text { regimens }\end{array}$ & GTR & $\begin{array}{c}1.8 \mathrm{~Gy} / 30.6 \mathrm{~Gy} \\
\text { to the tumor } \\
\text { bed }\end{array}$ & 0 & Bone \\
\hline 7 & 25 & Bone & 4321 & $\begin{array}{c}\mathrm{A} 9 \text { and } \mathrm{A} 11 \\
\text { regimens }\end{array}$ & GTR & $\begin{array}{c}1.5 \mathrm{~Gy} / 22.5 \mathrm{~Gy} \\
\text { to the tumor } \\
\text { bed }\end{array}$ & 14 & Bone \\
\hline
\end{tabular}

Abbreviations: LDH: Lactate dehydrogenase, Chemo: Chemotherapy; RT: Radiotherapy; GTR: Gross total resection; BM: Bone marrow; LMI: Leptomeningeal involvement.

*All but one patient (patient 6) initially treated with induction chemotherapy.

**Patient 3 also had autologous peripheral hematopoietic stem cell transplantation. 
Gultekin M. et al: Pediatric Neuroblastoma Patients with Brain Metastasis

Table II: Clinical Characteristics, Treatment Features, and Outcomes of NB Patients with Brain Metastases

\begin{tabular}{|c|c|c|c|c|c|c|}
\hline $\begin{array}{l}\text { Patient } \\
\text { no. }\end{array}$ & $\begin{array}{l}\text { No. of brain } \\
\text { metastasis }\end{array}$ & Metastatic site & Surgery & RT (field/dose) & Chemo & Outcome \\
\hline 1 & 1 & $\begin{array}{l}\text { Right parietal lobe } \\
\text { and LMI }\end{array}$ & GTR & $\begin{array}{c}\text { WBRT } \\
3 \text { Gy/30 Gy }\end{array}$ & VCR+CARB+CP & $\begin{array}{l}\text { Currently alive for } \\
164 \text { months }\end{array}$ \\
\hline 2 & 1 & Right parietal lobe & GTR & $\begin{array}{c}\text { WBRT } \\
1.8 \text { Gy/30.6 Gy }\end{array}$ & TPOG 2009 & $\begin{array}{l}\text { Currently alive for } \\
187 \text { months }\end{array}$ \\
\hline 4 & 1 & Left temporal lobe & No & $\begin{array}{l}\text { Metastatic lesion } \\
1.8 \mathrm{~Gy} / 36 \mathrm{~Gy}\end{array}$ & ICE & Died at 40 months \\
\hline 5 & 2 & $\begin{array}{l}\text { Left temporal lobe } \\
\text { and left frontal } \\
\text { lobe }\end{array}$ & STR & $\begin{array}{c}\text { WBRT and SIB } \\
\text { (metastatic lesion) } \\
1.92 \mathrm{~Gy} / 25 \mathrm{~Gy} \\
2.3 \mathrm{~Gy} / 30 \mathrm{~Gy}\end{array}$ & $\begin{array}{c}\mathrm{ICE}+\text { Irinotecan }+\mathrm{VCR}+ \\
\mathrm{TMZ}\end{array}$ & Died at 19 months \\
\hline 7 & 2 & $\begin{array}{l}\text { Left frontal lobe } \\
\text { and left temporal } \\
\text { lobe }\end{array}$ & No & $\begin{array}{l}\text { Metastatic lesion } \\
2.5 \mathrm{~Gy} / 30 \mathrm{~Gy}\end{array}$ & ICE & Died at 17 months \\
\hline
\end{tabular}

Abbreviations: No.: Number, Dx: Diagnosis, LMI: Leptomeningeal involvement, GTR: Gross total resection, STR: Subtotal resection, WBRT: Whole brain radiotherapy, SIB: Simultaneous integrated boost, CSI: Craniospinal irradiation, RT: Radiotherapy, Chemo: Chemotherapy, VCR: Vincristine, CARB: Carboplatin, CP: Cyclophosphamide, TPOG: Turkish Pediatric Oncology Group, ICE: Iphosphamide + cisplatin + etoposide, TMZ:Temozolomide.

7/120 (5.8\%). Six (86\%) patients were diagnosed with brain metastases during therapy or follow-up, and the remaining one $(14 \%)$ patient was diagnosed with brain metastases at the time of initial diagnosis. The median time interval between the initial diagnosis and the brain metastasis was 14 months (range, 0-28 months) for all patients, 12 months (range, 0-28 months) for deceased patients, and 23 months (range, 19-26 months) for living patients $(p=0.245)$. At the time of brain metastasis, three patients (patients 1-3) had a complete response for primary or metastatic sites, while the other patients had active bone metastasis at the time of brain metastasis.

Two patients (patients 3 and 7) were asymptomatic at the time of brain metastasis, and brain metastasis was first detected on MIBG scintigraphy. Leptomeningeal metastasis in both the brain and spine was detected in one patient (patient 6). On brain MRI, all lesions were contrast-enhanced, and hemorrhage was detected in three patients (patients 1, 4, and 6). Hydrocephalus and peritumoral edema were observed in two patients (patients 1 and 2), and gross total resection was performed in these patients to relieve symptoms.

All patients had parenchymal brain metastasis. Three (43\%) patients had single brain metastasis, two (28.5\%) had two lesions, and the other two (28.5\%) had multiple metastases. The calvarium was also involved in three patients (patients 4, 5, and 7), and leptomeningeal involvement was detected in three patients (patients 1,3, and 6). After the diagnosis of brain metastasis, three (43\%) patients were treated with intracranial surgery. Two (29\%) patients underwent right craniotomy and gross total resection using the microsurgical technique (patients 1 and 2). Patient 5 had two parenchymal brain metastases. Thus, the patient was placed in a supine position with the head rotated to the right and subjected to left temporal craniotomy. Gross total resection was performed using a microsurgical technique for left temporal lobe lesions, but surgery was not performed for left frontal lobe lesions, and this intervention was accepted as subtotal resection (14\%) (Figure 1A-D). Biopsy was not obtained in the other $4(57 \%)$ patients. Although patient 4 had a solitary brain lesion, surgery was not performed, and the patient received radiotherapy to the lesion. While craniospinal irradiation was performed in patient 6 who had leptomeningeal metastasis in both the brain and spine, local $(n=2)$ or whole-brain radiotherapy $(n=4)$ was applied to all other patients. Corticosteroids were administered to four patients (patients 2, 4, 5, and 7) to reduce edema and relieve symptoms during the treatment of brain metastasis. The treatment was well tolerated; headache and vomiting were seen as acute toxicity in one patient (patient 5), and combined pituitary hormone deficiency was seen as late toxicity in the same patient.

The median follow-up time after treatment for brain metastasis was 14 months (range, 3-187 months). After the treatment of brain metastasis, three patients (patients 1, 2, and 3) had a complete response, but four patients (patients $4,5,6$, and 7 ) 


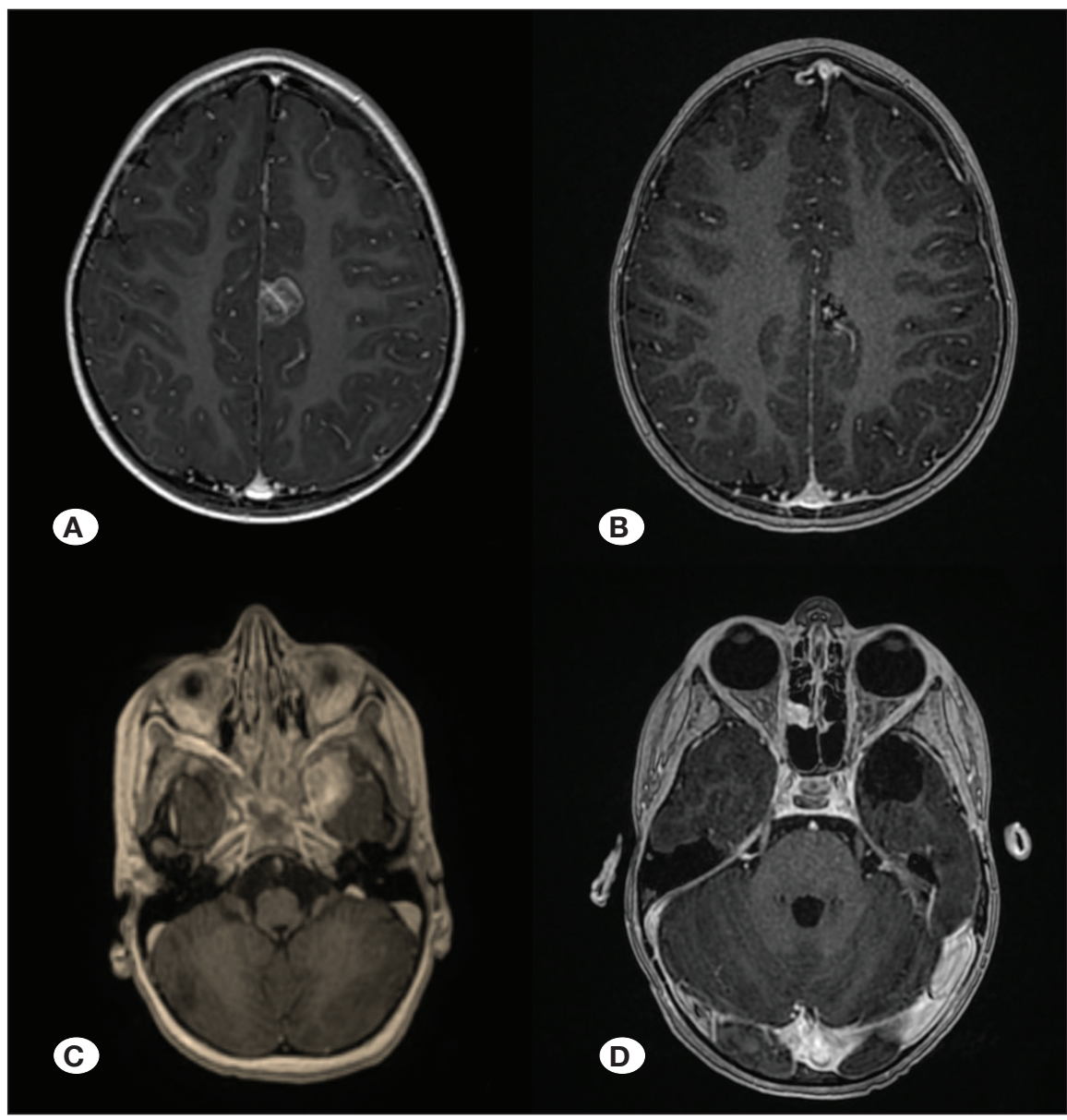

Figure 1: Axial MRI scans of patient 5. This patient had 2 brain lesions and gross total resection was performed for left temporal lobe lesion, but surgery was not performed for left frontal lobe lesion and this intervention was accepted as subtotal resection. A) Axial MRI scan of left frontal lobe lesion before radiotherapy. B) Axial MRI scan of left frontal lobe lesion 3 months after radiotherapy. C) Axial MRI scan of left temporal lobe lesion before surgery and radiotherapy. D) Axial MRI scan of left temporal lobe lesion 3 months after surgery and radiotherapy. had progressive disease in the brain. Although a complete response was initially achieved in patient 3 , this patient died 7 months after whole-brain radiotherapy due to progressive brain metastasis. Five patients died from progressive disease, and the remaining two patients were alive at 164 and 187 months, respectively. Gross total resection was performed in both patients, and relapse was not observed. In patient 5 , who underwent subtotal resection, a complete response was observed in the totally resected lesion, but the other lesion progressed, and leptomeningeal involvement occurred. All five patients who did not undergo surgery or underwent subtotal resection died from the progression of brain metastasis. The median overall survival time after the treatment of brain metastasis was $14 \pm 3.7$ months (7-22 months, 95\% Cl). While the 5 -year overall survival rate was $67 \%$ in patients with isolated brain metastases, the 5-year overall survival rate was $0 \%$ in the presence of other metastases $(p=0.221)$.

\section{DISCUSSION}

Brain metastases are rarely observed in childhood solid tumors (18). However, increased survival rates in patients with neuroblastoma due to advances in treatment modalities have led to more cases of brain metastasis during the remaining lifetime $(4,13,21,22)$. Our study revealed that the incidence of brain metastasis in patients with neuroblastoma was $5.8 \%$
(7/120), which is consistent with the findings of previous studies.

The differential diagnosis of brain lesions in pediatric patients should include medulloblastoma, ependymoma, poorly differentiated oligodendroglioma, brain metastasis, and primary central nervous system neuroblastoma. Primary central nervous system neuroblastoma is also rarely seen, similar to brain metastasis from neuroblastoma (8). Peri- and intraventricular lesions are frequent, and focal hemorrhage may occur (20). Although there is no pathognomonic appearance on $\mathrm{CT}$ or $\mathrm{MRI}$, histopathological confirmation is required for a definitive diagnosis (8). While patients with peripheral neuroblastoma characteristically have elevated levels of urine catecholamines, those with primary cerebral neuroblastoma have normal levels of urine catecholamines (3). Other possible sites for the primary tumor should also be investigated before a definitive diagnosis is made. In the current study, the primary tumor site was the adrenal gland in six patients and paraspinal region in one patient; levels of urine catecholamines were elevated in all patients; thus, all patients were diagnosed with brain metastasis from peripheral neuroblastoma.

Data regarding the risk factors and prognostic factors for brain metastasis in patients with neuroblastoma are controversial. Kramer et al. showed that a high LDH level (higher than $1500 \mathrm{U} / \mathrm{mL}$ ) was associated with an increased risk of brain 
metastasis (13). In contrast, Zhu et al. found no statistically significant difference in LDH levels between patients with and without brain metastasis (22). In the current study, two of seven patients had LDH levels higher than 1500 at the time of initial diagnosis. Both these patients died due to disease progression 17 and 30 months after brain metastases. Due to the low number of patients, we cannot draw conclusions about the possible prognostic and predictive effects of LDH in these patients.

The time interval between the initial diagnosis and the development of brain metastases may have prognostic value in determining survival and selecting the best treatment modality. In the current study, the median time intervals between the initial diagnosis and the date of brain metastasis in all, deceased, and alive patients were 14, 12, and 23 months, respectively. Although statistical significance was not observed, a shorter time interval between the initial diagnosis and the date of brain metastasis may be a negative prognostic factor for survival. Similar to our study, Kellie et al. also demonstrated that the time interval was 24 and 34 months in patients who were alive 23 and 62 months after the development of brain metastasis, respectively (12). In another study by $\mathrm{Hu}$ et al., the median time interval between the initial diagnosis and brain metastasis was 29.5 months, resulting in the longest survival time (median 40 months) in the literature (10).

Survival rates are poor in patients with neuroblastoma with brain metastasis. A literature review reported that the median survival time after the development of brain metastasis ranges from 1 to 14.1 months (13). The optimal treatment for brain metastasis has not been clearly defined in the literature. These patients are often treated with palliative radiotherapy and chemotherapy, and surgery is usually reserved for patients who need urgent decompression (19). In a recent retrospective study by $\mathrm{Hu}$ et al., it was shown that combined radiotherapy and/or autologous stem cell transplantation produced a statistically significantly longer overall survival time compared to chemotherapy and simple operation. In addition, patients treated with combined intracranial surgery and/or radiotherapy had significantly longer survival times than those treated with chemotherapy alone (10). The 2-year survival rate in this study was $82.2 \%$. The median overall survival time after treatment for brain metastasis was 14 months, and the 2-year overall survival rate was $57 \%$ in our study. The main difference between our study and that of $\mathrm{Hu}$ et al. was that all patients in our study were treated with radiotherapy in addition to chemotherapy and/or surgery, while 4 out of 15 patients received RT in Hu et al.'s study. All four patients in Hu et al.'s study were treated with craniospinal irradiation, while only one out of seven in our study was treated with craniospinal irradiation. The number of the metastases was $1.67 \pm 1.35$ in Hu et al.'s study, and it was $2.29 \pm 1.60$ in our study. Only three patients in our study had solitary brain metastases, and the 2 -year overall survival rate was $67 \%$ in these patients, which was similar to that reported by $\mathrm{Hu}$ et al.

Two patients with solitary brain metastasis in our study were treated with gross total resection, while one patient underwent subtotal resection before whole-brain radiotherapy. These two patients with gross total resection also had no other disease sites at the time of brain metastases and had no evidence of disease at 164 and 187 months of follow-up. A few studies have reported that patients with solitary brain metastasis who underwent surgery have prolonged survival compared to patients without surgery $(12,13,22)$. While the median survival time after the development of brain metastasis was 4 months in a study by Zhu et al., one patient who underwent complete surgical resection was still alive for 47 months (22). Similar to our study, patients who were treated with combined intracranial surgery and/or radiotherapy in Hu et al.'s study had a significantly longer survival time compared to those who underwent chemotherapy $(46.67 \pm 6.69$ vs. $16.42 \pm 1.42$ months) (10).

Although radiotherapy seems to be an indispensable part of the treatment of neuroblastoma patients with brain metastases, there is no agreement on the radiotherapy field and dose. While whole-brain radiotherapy is commonly applied as standard therapy, craniospinal irradiation should be performed in cases with cerebrospinal fluid positivity or leptomeningeal involvement. Kellie et al. reported the longest survival (62 months) in the literature with gross total resection and craniospinal irradiation (cranium 30 Gy, spine 20 Gy) in a neuroblastoma patient with brain metastasis without evidence of cerebrospinal fluid positivity (12). Although there is insufficient evidence for the application of prophylactic craniospinal irradiation, some authors have suggested craniospinal irradiation in high-risk patients for cerebrospinal fluid involvement (i.e., lumbar puncture at diagnosis) (13). Only one patient who had leptomeningeal dissemination in our study was treated with craniospinal irradiation and died 30 months after radiotherapy. All other patients were treated with wholebrain or local radiotherapy. Two patients who were treated with local radiotherapy to the metastatic lesion survived for only 17 and 40 months after radiotherapy. The other two patients treated with whole-brain radiotherapy survived for 7 and 19 months. The two special cases with solitary brain metastasis who underwent gross total resection followed by whole-brain radiotherapy (30 Gy) and chemotherapy were alive at 164 and 187 months, respectively. To the best of our knowledge, these are the longest survival rates reported in the literature. These findings support that the extent of disease per se, not the extent of radiotherapy, affects the outcome.

This study has some limitations owing to its retrospective nature. First, patients were treated over a long period, in which the treatment strategies could change over time. Second, we reported the treatment results of only seven neuroblastoma patients with brain metastasis; thus, we could not reach a definite result. However, all patients were treated at a single institute with a risk-based national neuroblastoma treatment protocol.

\section{CONCLUSION}

Brain metastasis of neuroblastoma is a rare entity, and the optimal treatment is not clearly defined. However, a combination of gross total resection, chemotherapy, and 
radiotherapy appears to improve patient survival. A short time interval between the initial diagnosis and brain metastasis, multiple brain metastases, and less than gross total resection of the tumor and additional metastatic sites other than the brain seem to be negative prognostic factors. Further multicenter studies with a large number of patients may clarify the prognostic factors of neuroblastoma patients with brain metastases, including the optimal treatment approach.

\section{REFERENCES}

1. Aksoylar S, Varan A, Vergin C, Hazar V, Akici F, Dagdemir A, Buyukavci M, Kebudi R, Kurucu N, Sevinir B, Unal E, Vural S, Guler E, Apak H, Oniz H, Karadeniz C, Canpolat C, Anak $\mathrm{S}$, Ilhan I, Ince D, Cecen E, Olgun N: Treatment of highrisk neuroblastoma: National protocol results of the Turkish Pediatric Oncology Group. J Cancer Res Ther 13:284-290, 2017

2. Astigarraga I, Lejarreta R, Navajas A, Fernandez-Teijeiro A, Imaz I, Bezanilla JL: Secondary central nervous system metastases in children with neuroblastoma. Med Pediatr Oncol 27:529-533, 1996

3. Berger MS, Edwards MS, Wara WM, Levin VA, Wilson CB: Primary cerebral neuroblastoma. Long-term follow-up review and therapeutic guidelines. J Neurosurg 59:418-423, 1983

4. Blatt J, Fitz C, Mirro J Jr: Recognition of central nervous system metastases in children with metastatic primary extracranial neuroblastoma. Pediatr Hematol Oncol 14:233241, 1997

5. Bouffet E, Doumi N, Thiesse P, Mottolese C, Jouvet A, Lacroze M, Carrie C, Frappaz D, Brunat-Mentigny M: Brain metastases in children with solid tumors. Cancer 79:403-410, 1997

6. Brodeur GM, Pritchard J, Berthold F, Carlsen NL, Castel V, Castelberry RP, De Bernardi B, Evans AE, Favrot M, Hedborg $\mathrm{F}$ : Revisions of the international criteria for neuroblastoma diagnosis, staging, and response to treatment. J Clin Oncol 11:1466-1477, 1993

7. Cox JD, Stetz J, Pajak TF: Toxicity criteria of the Radiation Therapy Oncology Group (RTOG) and the European Organization for Research and Treatment of Cancer (EORTC). Int J Radiat Oncol Biol Phys 31:1341-1346, 1995

8. Davis PC, Wichman RD, Takei Y, Hoffman JC Jr: Primary cerebral neuroblastoma: CT and MR findings in 12 cases. AJNR Am J Neuroradiol 11:115-120, 1990

9. Deutsch $\mathrm{M}$, Orlando $\mathrm{S}$, Wollman $\mathrm{M}$ : Radiotherapy for metastases to the brain in children. Med Pediatr Oncol 39:6062, 2002

10. Hu H, Zhang W, Huang D, Wang Y, Zhang Y, Yi Y, Liu A, Li J: Clinical characteristics, treatment and prognosis of paediatric patients with metastatic neuroblastoma to the brain. Clin Neurol Neurosurg 184:105372, 2019
11. Kebudi R, Ayan I, Gorgun O, Agaoglu FY, Vural S, Darendeliler $\mathrm{E}$ : Brain metastasis in pediatric extracranial solid tumors: Survey and literature review. J Neurooncol 71:43-48, 2005

12. Kellie SJ, Hayes FA, Bowman L, Kovnar EH, Langston J, Jenkins JJ, 3rd, Pao WJ, Ducos R, Green AA: Primary extracranial neuroblastoma with central nervous system metastases characterization by clinicopathologic findings and neuroimaging. Cancer 68:1999-2006, 1991

13. Kramer K, Kushner B, Heller G, Cheung NK: Neuroblastoma metastatic to the central nervous system. The memorial sloankettering cancer center experience and a literature review. Cancer 91:1510-1519, 2001

14. Kutluk TYM: Turkish national pediatric cancer registry 2002-2008 (Turkish Pediatric Oncology Group and Turkish Pediatric Hematology Society). Pediatr Blood Cancer 53:851, 2009

15. Maris JM: Recent advances in neuroblastoma. N Engl J Med 362:2202-2211, 2010

16. Matthay KK, Brisse H, Couanet D, Couturier J, Benard J, Mosseri V, Edeline V, Lumbroso J, Valteau-Couanet D, Michon $\mathrm{J}$ : Central nervous system metastases in neuroblastoma: Radiologic, clinical, and biologic features in 23 patients. Cancer 98:155-165, 2003

17. Osawa S, Kumabe T, Saito R, Sonoda Y, Niizuma H, Watanabe $\mathrm{M}$, Tominaga $\mathrm{T}$ : Infratentorial brain metastases of pediatric non-epithelial malignant tumors: Three case reports. Brain Tumor Pathol 28:167-174, 2011

18. Paulino AC, Nguyen TX, Barker JL Jr: Brain metastasis in children with sarcoma, neuroblastoma, and Wilms' tumor. Int J Radiat Oncol Biol Phys 57:177-183, 2003

19. Schodel P, Schebesch KM, Brawanski A, Proescholdt MA: Surgical resection of brain metastases-impact on neurological outcome. Int J Mol Sci 14:8708-8718, 2013

20. Yaris N, Yavuz MN, Reis A, Yavuz AA, Okten A: Primary cerebral neuroblastoma: A case treated with adjuvant chemotherapy and radiotherapy. Turk J Pediatr 46:182-185, 2004

21. Zage PE, Kletzel M, Murray K, Marcus R, Castleberry R, Zhang Y, London WB, Kretschmar C, Children's Oncology G: Outcomes of the POG 9340/9341/9342 trials for children with high-risk neuroblastoma: A report from the Children's Oncology Group. Pediatr Blood Cancer 51:747-753, 2008

22. Zhu J, Wang J, Zhen ZJ, Lu SY, Zhang F, Sun FF, Li PF, Huang JT, Cai $R Q$, Sun XF: Brain metastasis in children with stage 4 neuroblastoma after multidisciplinary treatment. Chin $\mathrm{J}$ Cancer 34:531-537, 2015 